\title{
Highly Stable Mesoporous Ge@C Sphere Anodes for Lithium-ion Batteries
}

\author{
Chen Cheng ${ }^{\mathrm{a}}$, Jing $\mathrm{Xu}^{\mathrm{b*}}$ and Kai Xie \\ National University of Defense Technology, Changsha, 410073, China

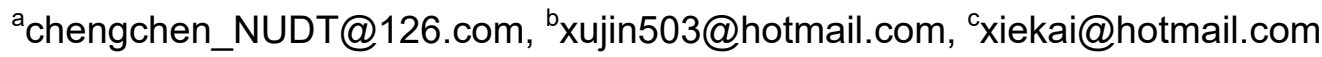

Keywords: highly stable; mesoporous; germanium; anode; lithium-ion batteries

\begin{abstract}
The Mesoporous Ge@C sphere has been designed via a modified Stöber method and an in-situ solution deposition. Compared with the pure Ge nanoparticles, the as-synthesized MesoporousGe@C sphere nanocomposites show much better cycling stability and rate capability. That as-prepared anode material can maintain a reversible capacity of $708.8 \mathrm{mAhg}^{-1}$ after 200 cycles at a rate of $0.2 \mathrm{C}$, and even at $5 \mathrm{C}$, it also exhibits a high discharge capacity of $\sim 522.4 \mathrm{mAhg}^{-1}$. The superior comprehensive electrochemical performance of the electrode is ascribed to the synergic effects of the porous structure and hollow carbon sphere, which can effectively alleviate the structural strain and suppress large volume changes, additionally, can reduce electrolyte diffusion path length beneficial to allow rapid electron transportation and let more $\mathrm{Li}^{+}$flux across the interface.
\end{abstract}

\section{Introduction}

Of late, high energy density and long cycle life lithium-ion batteries (LIBs) are wildly applied in electric vehicles driven by a rising demand for new energy vehicles. Extensive research has been devoted to developing new electrode materials capable of delivering high energy density and cycling stability as alternates to traditional graphite electrodes[1-3]. Germanium (Ge) has been considered as promising anode materials for LIBs due to a high theoretical capacity of $1600 \mathrm{mAhg}^{-1}$ and a superior intrinsic kinetics (high electrical conductivity and Li diffusion coefficient), making it more appropriate for high-power Li-ion batteries.

However, Ge suffers tremendous volume expansion during the Li-ion insertion and extraction processes, resulting in pulverization of the electrodes and degrading the capacity consequently. Fortunately, it has documented that nanoscale Ge with variously designed morphologies could improve cycling stability and rate capability because of its shorter lithium-diffusion distance and more reaction sites, such as nanoparticles[4], nanowires[5, 6], nanotubes[7]. Other alternate approaches to increase cyclability involve porous structure[8,9] and hybridizing with carbon materials[10, 11] or other components[12]. Over the last few years, large amounts of work combining germanium with carbon materials or other components are reported. Xiu Li et al[9] reported porous amorphous $\mathrm{Ge} / \mathrm{C}$ composites, which can deliver a reversible capacity of $681 \mathrm{mAhg}^{-1}$ after 350 cycles. Xuanwen Gao et al[12] prepared Nano-Germanium/polypyrrole composites. The electrode exhibits a lithium storage capacity of $1014 \mathrm{mAhg}^{-1}$ after 50 cycles at $0.2 \mathrm{C}$ rate.

Herein, we proposed mesoporous Ge@C sphere composites through a modified stöber method[13] and an in-suit solution deposition[14]. When used as anode material, the mesoporous Ge@C sphere electrode exhibits high cycling stability and superior rate capability. The mesoporous Ge@C sphere composites could maintain a reversible capacity of $708.8 \mathrm{mAhg}^{-1}$ after 200 cycles at a rate of $0.2 \mathrm{C}$, and even at $5 \mathrm{C}$, it also exhibits a high discharge capacity of $\sim 522.4 \mathrm{mAhg}^{-1}$. 


\section{Result and Discussion}

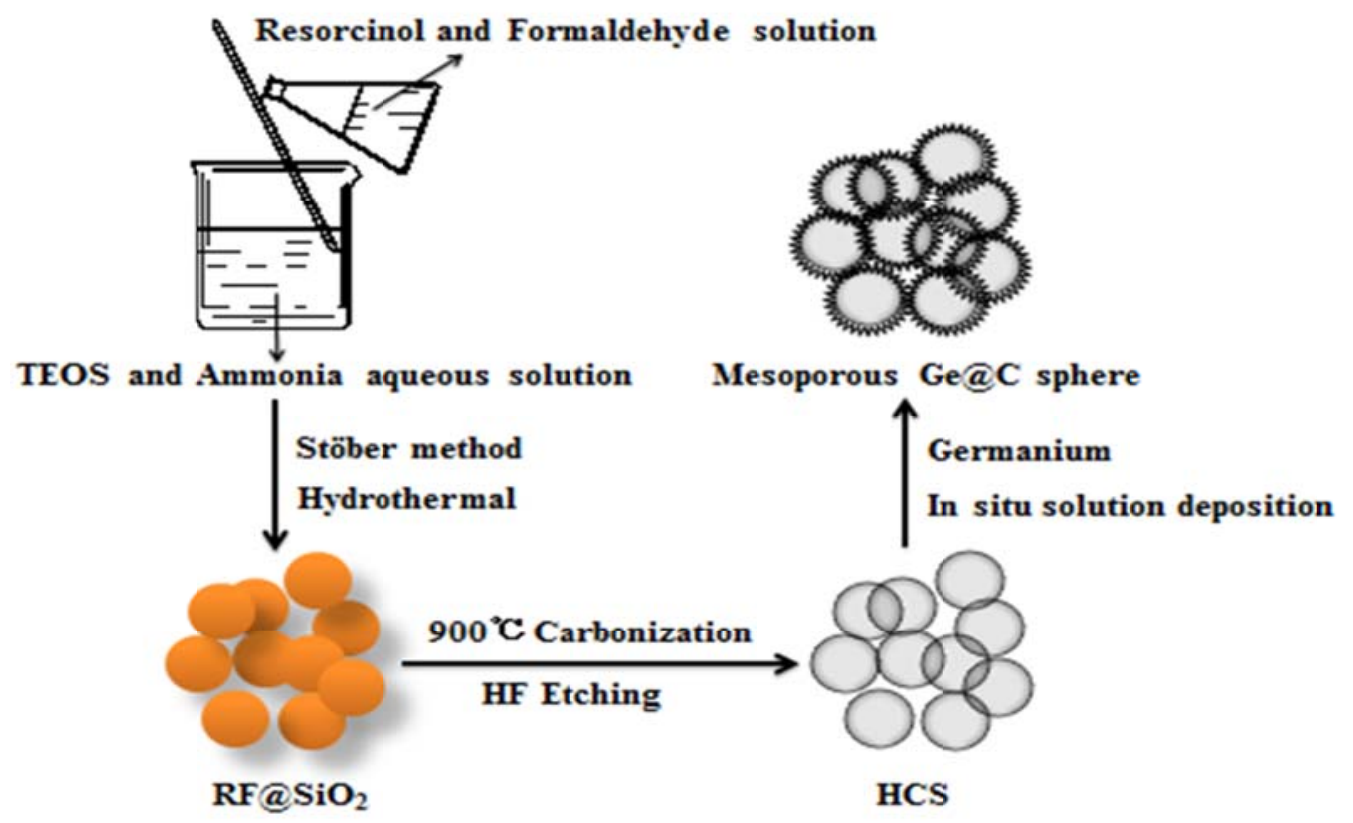

Scheme1. Schematic illustration of the synthesis route for the mesoporousGe@C sphere

The synthesized route for the mesoporous Ge@C sphere was shown in Scheme1. RF@ $\mathrm{SiO}_{2}$ nanospheres were prepared using a modified Stöber method (a typical process shown in the first step). Consequently, $\mathrm{RF} @ \mathrm{SiO}_{2}$ transformed to hollow carbon sphere (HCS) through the carbonization of $\mathrm{RF}$ resin at $900^{\circ} \mathrm{C}$ for $2 \mathrm{~h}$ and etching $\mathrm{SiO}_{2}$ with $\mathrm{HF}$ overnight. Importantly, the nanopores on the surface of HCSs were introduced by the HF etching process. At last, a mesoporous Ge@C sphere was synthesized by an in-situ solution deposition method. Ge was introduced by the reduction of $\mathrm{GeO}_{2}$ precursor, and then deposited on the surface of HCSs.
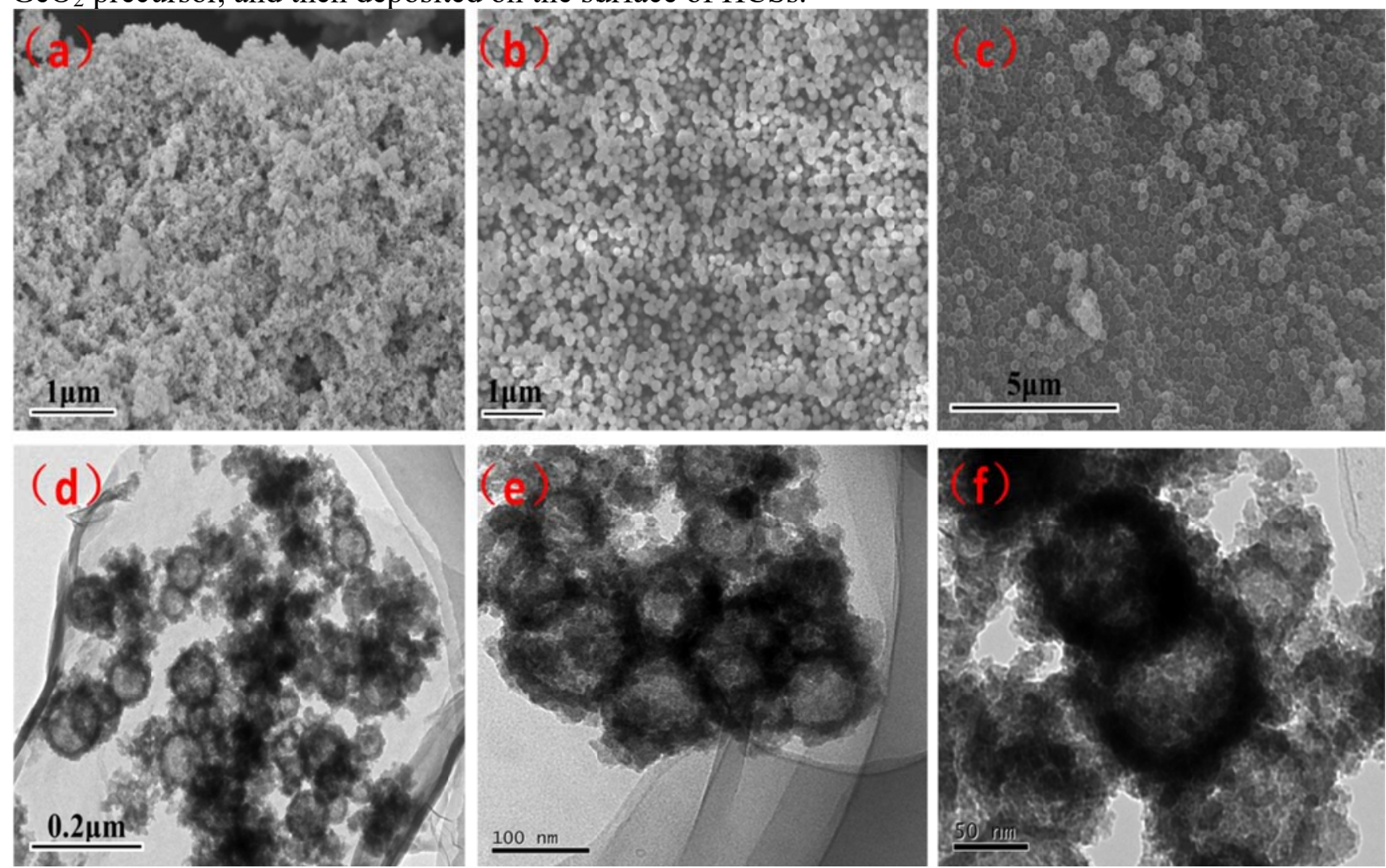

Figure.1 SEM images of (a) Ge nanoparticles, (b) RF@ $\mathrm{SiO}_{2}$ nanospheres, (c) HCS and (d)(e)(f) TEM images of the mesoporous Ge@C sphere 
Fig. 1a reveals the pristine Ge particle size is extremely small and these primary particles have clustered into large agglomerates. The smooth surfaces nanospheres with diameters of about 100 130 nm uniformly dispersed, shown in Fig.1b. After the carbonization and etching process, the HCS has a slightly shrunken diameter compared to RF@SiO2 nanospheres and its surface becomes rough, due to the pyrolysis of the RF resins during high temperature calcination (Fig.1c). Fig.1d,e and $\mathrm{f}$ exhibit the TEM images of the mesoporous Ge@C sphere in different magnifications. Simultaneously, it gave visible evidence that the Ge nanoparticles uniformly cover on the surface of the HCSs. The HCSs could not only act as a barrier to reduce the severe aggregation of Ge nanoparticles during charge/discharge processes, but also could be beneficial to allow rapid electron transportation due to the improved conductivity of the whole electrodes. Additionally, the HCSs could effectively alleviate the structural strain and suppress large volume changes during the cycling processes. Owing to the synergistic effect of the HCSs and nano-sizes, the mesoporous Ge@C sphere anodes exhibit a high-capacity, high-rate and long-life performance.
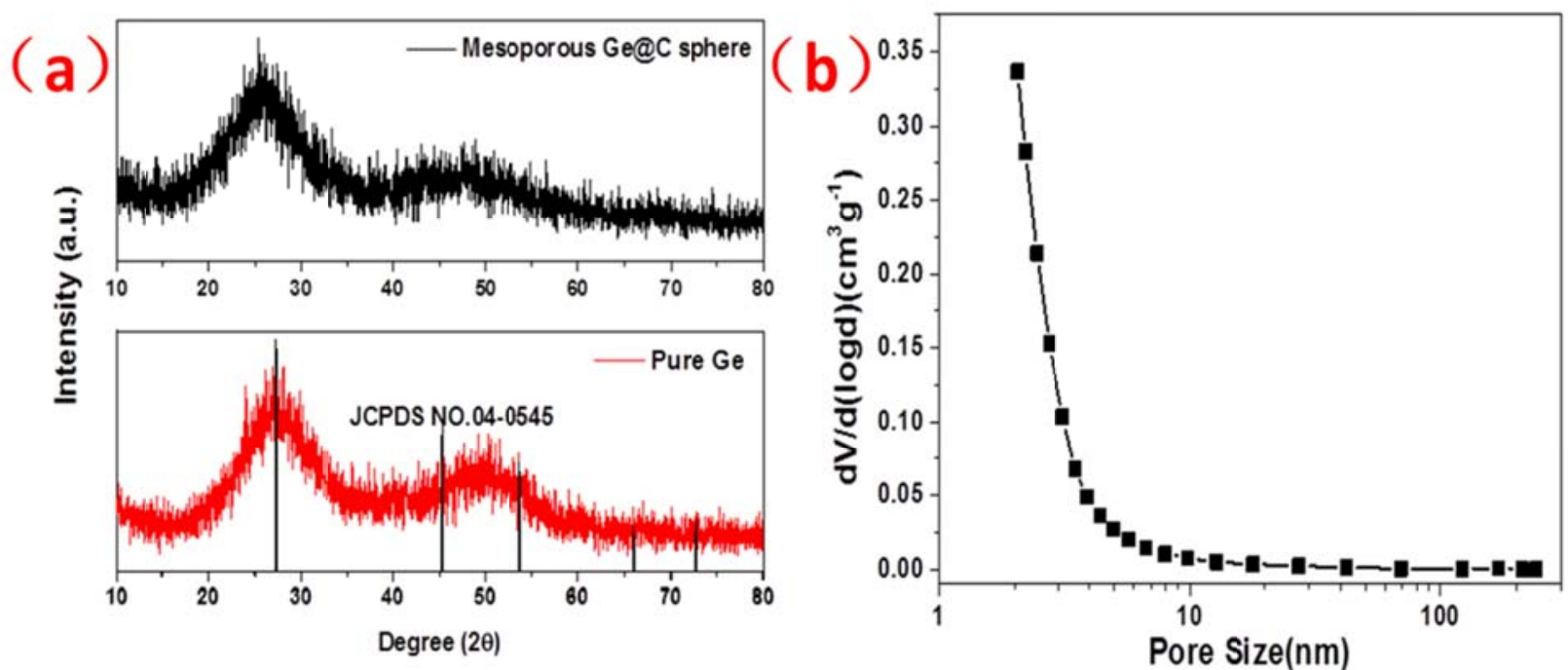

Figure.2 (a) XRD patterns of the Mesoporous Ge@C sphere and Pure Ge, (b) pore-size distribution of the Mesoporous Ge@C sphere

The X-ray diffraction (XRD) patterns of the as-synthesized pure Ge nanoparticles and mesoporous Ge@C sphere are presented in Fig.2a. Two broad diffraction peaks were present in the diffraction pattern of the pure Ge, suggesting the amorphous structure of $\mathrm{Ge}$, which could be indexed to the standard cubic phase Ge (JCPDS No. 04-545). Simultaneously, the diffraction peaks of mesoporous Ge@C sphere were similar to that of pure Ge. However, there was no diffraction peak for carbon in the nanocomposites, which might be ascribed to the broad diffraction peaks overlapping the diffraction peak for carbon. In Fig.2b, the pore size distribution curve suggests that the nanocomposites have a large amount of mesoporous (the porous size ranging from $2 \mathrm{~nm}$ to $10 \mathrm{~nm}$ ), which can mitigate the mechanical stress and buffer the volume changes during cycling. 

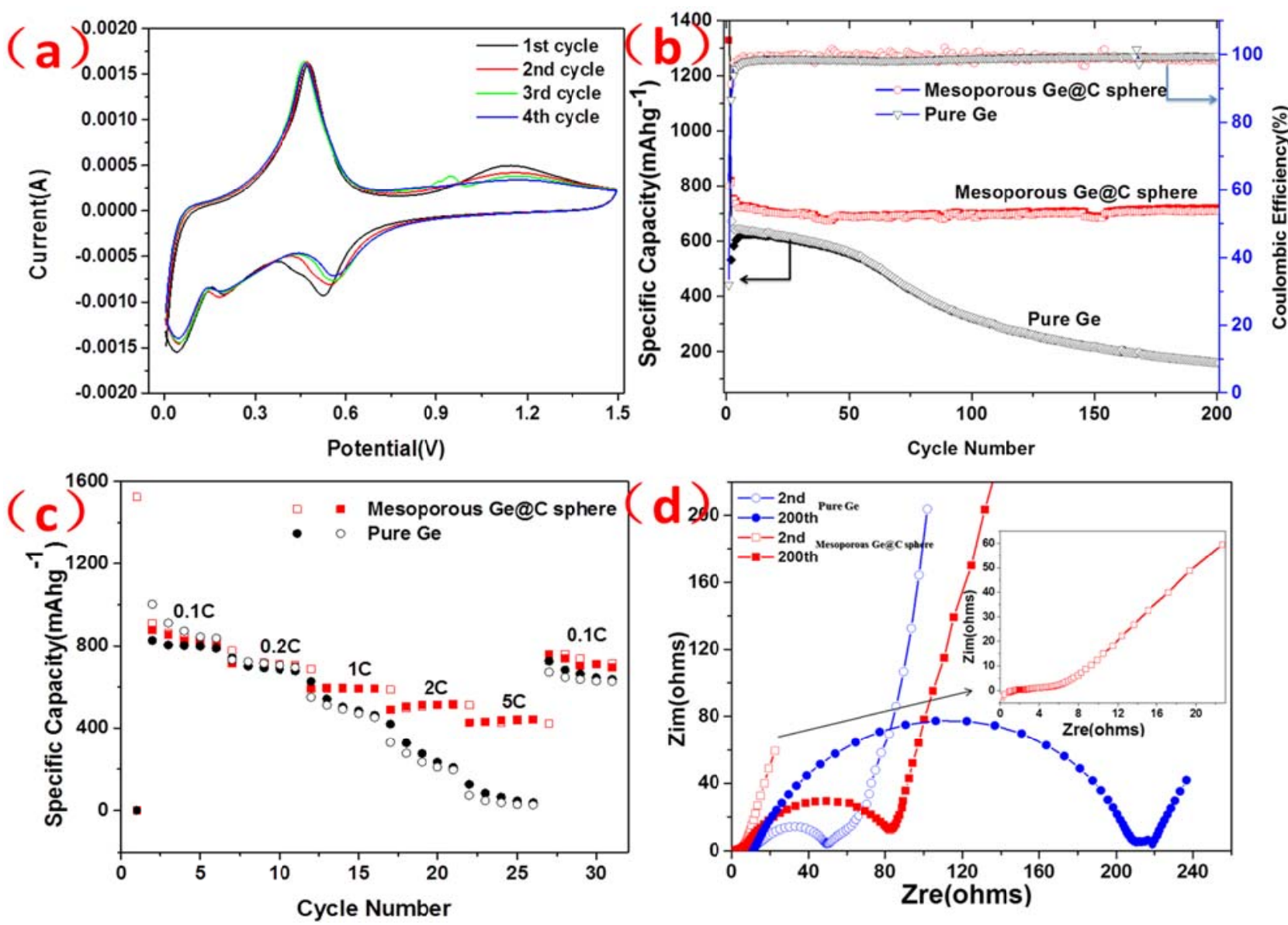

Figure.3 Electrochemical tests of the electrode (a) cyclic voltammograms of the Mesoporous Ge@C sphere; (b) the cycling performance and the corresponding coulombic efficiency of the Mesoporous $\mathrm{Ge} @ \mathrm{C}$ sphere and pure Ge at $0.2 \mathrm{C}$; (c) the specific capacity at different rates of the two electrode;

(d) Nyquist plots of the two electrodes at different cycles

Fig.3a shows the first four cyclic voltammetry (CV) curves of the Mesoporous Ge@C sphere electrode. Except for the little peak shift, the four CV curves overlapped with each other, which indicates the excellent reversibility and stability of the electrode. Fig. $3 b$ exhibits the cycling performance and the corresponding coulombic efficiency of the Mesoporous Ge@C sphere and pure $\mathrm{Ge}$ at $0.2 \mathrm{C}\left(1 \mathrm{C}=1600 \mathrm{mAhg}^{-1}\right)$. The two electrodes deliver very high first charge/discharge capacities of $532.2 \mathrm{mAhg}^{-1} / 1668.2 \mathrm{mAhg}^{-1}$ and $813.9 \mathrm{mAhg}^{-1} / 1329.9 \mathrm{mAhg}^{-1}$ with an initial columbic efficiency of $31.9 \%$ and $61.2 \%$, respectively. The dramatic capacity loss was observed in the first 5 cycles, which was attributed to the formation of solid electrolyte interface (SEI) films. However, the Mesoporous Ge@C sphere electrode remains highly stable in the subsequent cycles and delivers a high reversible specific capacity of $708.8 \mathrm{mAhg}^{-1}$ after 200 cycles. For a comparison, the dramatic capacity fading of the pure Ge electrode existed throughout the 200cycles and only a specific capacity of $159.4 \mathrm{mAhg}^{-1}$ was reserved.

Fig.3c reveals the specific capacity of the Mesoporous Ge@C sphere electrode was about 910.0, $776.5,688.3,587.2$ and $522.4 \mathrm{mAhg}^{-1}$ at current rate from $0.1,0.2,1,2$ to $5 \mathrm{C}$. When the rate goes back to $0.1 \mathrm{C}$, the specific capacity of the Mesoporous Ge@Csphere anode reversibly recovers to $757.2 \mathrm{mAhg}^{-1}$, demonstrating superior electrochemical reversibility. However, after the high-rate measurements, the pure Ge delivers very low specific capacity of $72.9 \mathrm{mAhg}^{-1}$, caused by the Ge nanoparticles lost contact to $\mathrm{Cu}$ collector in electrode pulverization process. Fig.3d displays Nyquist plots of the two electrodes at different cycles. After 200 cycles, the charge transfer resistance of the Mesoporous Ge@Csphere anode increased from $6 \Omega$ (as shown in the inset of Fig.3d) to $80 \Omega$, much lower than that of pure $\mathrm{Ge}$ (from $50 \Omega$ to $220 \Omega$ ). Low charge transfer resistance is beneficial to $\mathrm{Li}^{+}$ transfer at the interface of the electrolyte/electrode for the good cycle performances. 


\section{Conclusion}

In conclusion, the Mesoporous Ge@Csphere was synthesized via a modified Stöber method and an in-situ solution deposition. When used as anode material, the mesoporous Ge@C sphere electrode exhibits high cycling stability and superior rate capability. The mesoporous Ge@C sphere composites could maintain a reversible capacity of $708.8 \mathrm{mAhg}^{-1}$ after 200 cycles at a rate of $0.2 \mathrm{C}$, and even at $5 \mathrm{C}$, it also exhibits a high discharge capacity of $\sim 522.4 \mathrm{mAhg}^{-1}$.

\section{References}

[1] M. Armand, J.M. Tarascon, Building better batteries, Nature, 451 (2008) 652-657.

[2] J.B. Goodenough, Y. Kim, Challenges for Rechargeable Li Batteries $\uparrow$, Chem. Mater., 22 (2010) 587-603.

[3] Peter, \#x, G.B. Prof., B. Scrosati, J.M. Tarascon, Nanomaterials for Rechargeable Lithium Batteries †, Angew. Chem., 47 (2008) 2930-2946.

[4] S. Jin, L. Na, C. Hao, C. Wang, Embedded into Graphene Ge Nanoparticles Highly Dispersed on Vertically Aligned Graphene with Excellent Electrochemical Performance for Lithium Storage, ACS Appl. Mater. Interfaces, 6 (2014) 19397-19404.

[5] T. Kennedy, E. Mullane, H. Geaney, M. Osiak, C. O'Dwyer, K.M. Ryan, High-performance germanium nanowire-based lithium-ion battery anodes extending over 1000 cycles through in situ formation of a continuous porous network, Nano Lett., 14 (2014) 716-723.

[6] X. Liu, J.Y. Huang, H. Shan, S.T. Picraux, J. Li, T. Zhu, Reversible nanopore formation in Ge nanowires during lithiation-delithiation cycling: an in situ TEM study, Nano Lett., (2011).

[7] G. Cui, G. Lin, N. Kaskhedikar, P.A.V. Aken, J. Maier, A novel germanium/carbon nanotubes nanocomposite for lithium storage material is, Electrochim. Acta, 55 (2010) 985-988.

[8] C. Xiao, Ge@C three-dimensional porous particles as high-performance anode materials of lithium-ion batteries, Rsc Advances, 5 (2015) 63056-63062.

[9] X. Li, W. Guo, Q. Wan, J. Ma, Porous amorphous Ge/C composites with excellent electrochemical properties, Rsc Advances, 5 (2015) 28111-28114.

[10] G. Cui, L. Gu, L. Zhi, N. Kaskhedikar, P.A. Van Aken, K. Müllen, J. Maier, A GermaniumCarbon Nanocomposite Material for Lithium Batteries, Adv. Mater., 20 (2008) 3079-3083.

[11] D.J. Xue, S. Xin, Y. Yan, K.C. Jiang, Y.X. Yin, Y.G. Guo, L.J. Wan, Improving the electrode performance of Ge through Ge@C core-shell nanoparticles and graphene networks, J. Am. Chem. Soc., 134 (2012) 2512-2515.

[12] X. Gao, W. Luo, C. Zhong, D. Wexler, S.L. Chou, H.K. Liu, Z. Shi, G. Chen, K. Ozawa, J.Z. Wang, Novel germanium/polypyrrole composite for high power lithium-ion batteries, Scientific Reports, 4 (2014) 6095-6095.

[13] D.J. Liu, P.S.Z. Qiao, D.H. Liu, D.J. Chen, A. Orpe, P.D. Zhao, P.G.Q. Lu, Extension of The Stöber Method to the Preparation of Monodisperse Resorcinol-Formaldehyde Resin Polymer and Carbon Spheres $\uparrow$, Angewandte Chemie International Edition, 50 (2011) 3336-3341.

[14] J. Wu, Y. Sun, R. Zou, G. Song, Z. Chen, C. Wang, J. Hu, One-step aqueous solution synthesis of Ge nanocrystals from GeO2 powders, CrystEngComm, 13 (2011) 3674-3677. 